\title{
Meningitis due to Fusobacterium necrophorum
}

\author{
Luana Coltella, Paola Bernaschi, Cristina Russo, Livia Mancinelli, Donato Menichella \\ U.O.C. di Microbiologia, Dipartimento dei Laboratori, Ospedale Pediatrico Bambino Gesù-IRCCS, Roma
}

Key words: F. necrophorum, Lemierre syndrome, meningitis

Meningite da Fusobacterium necrophorum

\section{SUMMARY}

Introduction: Fusobacterium necrophorum is an anaerobic Gram negative bacillus highly virulent, responsible, usually in children or adolescents, of localized abscesses and pharynx, as well as severe systemic infections, called Lemierre syndrome.

Methods: A I 5 year old child came to the emergency department (ED) of Bambino Gesù Children Hospital. Physicians prescribed chemicalphysical examination on blood and liquor, blood cultures for aerobic and anaerobic bacteria and for fungi (BD Ped Plus, lytic Ana, Micosis), microbiological culture on liquor (CSF) and on the swab from the right outer ear.

Results: On chemical examination, liquor appears cloudy, with $309 \mathrm{mg} / \mathrm{dl}$ of total proteins, glucose undetectable, WBC II, $160 \mathrm{mmc}, 92 \%$ of neutrophils. Hyperleukocytosis was detected also on the emocrome (WBC $21 \times 103 / \mu \mathrm{l}, 92 \%$ neutrophils). No bacterial antigens were detected. CSF culture resulted negative for aerobic bacteria, even after 48 hours of incubation. After 24 hours of inoculation, the blood culture for anaerobic bacteria resulted positive and, Fusobacterium necrophorum was isolated and identified, by genomic sequencing, after 24 hours growth on Schaedler medium. Microbiological culture of the right outer ear swab, highlighted only Corynebacterium spp. After 6 days from admission, the patient died for meningitis.

Conclusion:This event has shown the severity of infection by F. necrophorum and, at the same time, the underestimation of this germ in the spectrum of etiologic agents responsible for meningitis. The only microbiological indication was obtained from the anaerobes bacteria blood culture. Following this episode our working procedures for what concerns liquor samples management was modified, including routinely the investigation for anaerobic bacteria. Presumably this episode of meningitis has originated from a $F$. necrophorum otitis of the right ear, unfortunately not microbiologically confirmed. The anaerobic bacteria should always be considered as potentially responsible of meningitis, especially when ear infections or pharyngeal abscesses precede or accompany the onset of symptoms.

\section{INTRODUZIONE}

Fusobacterium necrophorum è un bacillo anaerobio Gram negativo inizialmente descritto negli animali, dove rappresenta un patogeno piuttosto comune ed estremamente virulento. Nell'uomo è responsabile di infezioni localizzate, principalmente a carico del distretto oro-faringeo e peritonsillare (10\% delle faringiti acute e $21 \%$ di quelle ricorrenti (1)) e di gravi infezioni sistemiche note come necrobacillosi o "sindrome di Lemierre” (2). Tale malattia ha origine da un'infezione nella regione testa-collo, nell' $87 \%$ dei casi si tratta di una faringite (1), ma può anche svilupparsi in seguito ad otiti, mastoiditi, sinusiti o parotiti. Durante l'infezione primaria $F$. necrophorum colonizza il sito di infezione e, in seguito ad alterazioni della mucosa faringea e alla reazione infiammatoria locale, viene diffuso per via ematica ad altre sedi. Il quadro classico di una malattia invasiva da F. necrophorum, come descritto da Lemierre, è quello di un giovane adulto o adolescente con una storia di faringite o mal di gola seguita da febbre alta e rigidità, che hanno inizio il $4^{\circ} 05^{\circ}$ giorno dopo i sintomi alla gola. A questo si accompagna linfoadenopatia cervicale e solitamente tromboflebite della vena giugulare interna. Le condizioni del paziente spesso peggiorano fino al coma e, infezioni non trattate, portano a morte entro 7-15 giorni.

Secondo Lemierre la malattia segue un decorso così caratteristico che è "praticamente impossibile non identificarla", tuttavia, mentre agli inizi del $\mathrm{XX}^{\circ}$ secolo era abbastanza comune, dopo la scoperta della penicillina è diventata una malattia piuttosto rara, e di conseguenza poco considerata, fino ad essere definita "the forgotten disease". Agli inizi degli anni '90, tuttavia, la riduzione nell'uso di antibiotici può aver contribuito ad un nuovo incremento (3).

\section{CASO CLINICO}

Un ragazzo di 15 anni, con febbre alta persistente e con pregressa otite dell'orecchio destro, giunge al Dipartimento di Emergenza (DEA) del Bambino Gesù inviato dal medico curante per sospetta meningite.

I genitori riferiscono che il ragazzo ha febbre alta da 2 gg, dal giorno precedente presenta sonnolenza, nella notte ha avuto otalgia e un picco febbrile. All'esame obiettivo le condizioni generali vengono definite gravi: il ragazzo al momento dell'accettazione non ha febbre, presenta cute pallida, sudorazione algida, rigidità nucale, viene evidenziata una secrezione purulenta con striature ematiche che fuoriesce dal condotto uditivo esterno destro.

Viene eseguita una puntura lombare, il liquor appare torbido, la pressione in ingresso è di $7.5 \mathrm{mmHg}$, in uscita è di 2 mmHg. Dal DEA vengono richiesti: emocromo, esame chimico-microscopico del liquor, esame batterioscopico e liquorcoltura, ricerca antigeni batterici su liquor, emocoltura per aerobi, anaerobi e miceti (BD Ped Plus, Lytic Ana, Micosis), esame colturale su tampone dell'orecchio destro. La terapia somministrata è ceftriaxone 3.5 gr e BBK8 $450 \mathrm{mg}$.

Poche ore dopo il prelievo $\left(\mathrm{T}_{0}\right)$ vengono comunicati l'emocromo, l'esame chimico-microscopico del Liquor, gli antigeni batterici e l'esame batterioscopico.

Emocromo: WBC 20.81 x 103/uL, Neutrofili 91.9\%, Linfociti $2.9 \%$, Proteina C reattiva $31.96 \mathrm{mg} / \mathrm{dl}$.

Esame chimico-microscopico del liquor: aspetto torbido, colore lattescente, proteine totali $309 \mathrm{mg} / \mathrm{dl}$, glucosio indosabile, cloruri 124 mmlo/L, WBC 11.160 mmc con 92\% di neutrofili. Antigeni batterici su Liquor (Wellcogen Bacteria Antigen kit, Unipath Oxoid S.p.A.): negativi per $H$. influenzae tipo b, $S$. pneumoniae, E. coli k1, Streptococcus gruppo B, N. meningitidis gruppo A, B, C, Y e W135.

Esame batterioscopico del Liquor: negativo, ma viene messa in evidenza la presenza di un tappeto di cellule.

La diagnosi chimico-clinica è di meningite (batterica).

Dopo 24h il flacone emocoltura per anaerobi viene rilevato positivo e all'esame batterioscopico si evidenziano bacilli Gram negativi. Vengono allestite, secondo procedura, le subcolture per la ricerca di germi aerobi, anaerobi e miceti e dopo $24 \mathrm{~h}$ (48h dal prelievo) l'unica sub-coltura positiva risulta quella per germi anaerobi. La diagnosi preliminare è di meningite da bacilli Gram negativi anaerobi obbligati, confermata anche dalla prova di aerotolleranza.

Dopo $72 \mathrm{~h}$ dal prelievo viene comunicata al reparto l'identifi- 
cazione definitiva dell'agente eziologico, Fusobacterium necrophorum, ottenuta mediante sequenziamento genico di 500bp del gene 16S rDNA (MicroSeq 500 Apllied Biosystems) e il relativo antibiogramma (Tabella 1), eseguito, come da linee guida CLSI, con metodica E-test. Il germe risulta sensibile solo a Metronidazolo e Clindamicina e, tra le altre resistenze, presenta quella al Cefotaxime, cefalosporina di terza generazione analoga del Ceftriaxone, usato nelle prime $72 \mathrm{~h}$ come terapia antibiotica in aggiunta al BBK8.

L'esame colturale del liquor risulta negativo anche dopo $48 \mathrm{~h}$ e dopo arricchimento in brodo.

L'esame colturale del tampone dell'orecchio esterno destro, eseguito come da richiesta per la ricerca di germi aerobi e miceti, presenta solo la crescita di un saprofita, Corynebacterium spp.

Dopo 6 giorni dal ricovero il paziente è deceduto in seguito a meningite.

In Figura I è schematizzato il percorso diagnostico con la relativa tempistica.

Tabella I. Antibiogramma del F. necrophorum isolato.

\begin{tabular}{lcc}
\hline Antibiotico & MIC $(\mu \mathrm{g} / \mathbf{m l})$ & Interpretazione \\
\hline Penicillina G (PG) & $>=32$ & $\mathrm{R}$ \\
\hline Amoxicillina/Acido Clavulanico $(\mathrm{XL})$ & $>=256$ & $\mathrm{R}$ \\
\hline Cefotaxime (CT) & $>=32$ & $\mathrm{R}$ \\
\hline Imipenem (IP) & $>=32$ & $\mathrm{R}$ \\
\hline Metronidazolo (MZ) & 0,25 & $\mathrm{~S}$ \\
\hline Clindamicina (CM) & 0,032 & $\mathrm{~S}$ \\
\hline
\end{tabular}

\section{DISCUSSIONE}

Questo episodio mostra la severità di un'infezione da $F$. necrophorum e allo stesso tempo la mancata considerazione di questo germe nello spettro degli agenti eziologici responsabili di meningiti.

Dagli esami di screening chimico fisico era emersa una prima indicazione di possibile infezione batterica (Proteina $\mathrm{C}$ reattiva elevata, 92\% di neutrofili).

La diagnosi di meningite da $F$. necrophorum è avvenuta solo indirettamente dall'emocoltura positiva, poiché l'esame colturale standard del Liquido cerebrospinale non includeva, di routine, l'uso di terreni specifici per germi anaerobi. La ricerca dei germi anaerobi nei campioni di Liquor era stata prevista a parte, per quei casi con un quadro clinico riconducibile ad una meningite da batteri anaerobi.

Anche l'esame colturale del materiale purulento prelevato dall'orecchio destro non ha permesso l'isolamento del germe responsabile dell'otite/meningite, perché al momento della richiesta il prelievo è stato definito "superficiale", e pertanto non ha incluso la ricerca di default dei germi anaerobi.

Nell'ipotesi eziologica iniziale sono stati inclusi soltanto germi patogeni aerobi; l’unica indagine per germi anaerobi è stata rappresentata dall'emocoltura specifica. Dopo 48h il microbiologo è stato in grado di fornire un'indicazione specifica di infezione sistemica sostenuta da bacilli Gram negativi anaerobi, permettendo un trattamento terapeutico mirato del paziente.

\section{CONCLUSIONI}

La coltura del Liquido cerebrospinale, di routine, non includeva l'uso di terreni specifici per germi anaerobi. In seguito a questo episodio abbiamo modificato le nostre procedure operative includendo nel pannello di valutazione microbiologica dei campioni di Liquor anche la ricerca per germi anaerobi. Presumibilmente questo episodio di meningite da F. necrophorum ha avuto origine da un'otite suppurativa dell'orecchio destro, purtroppo manca la conferma dell'esame colturale perché non è stata richiesta la ricerca per germi anaerobi.

Anche se una malattia invasiva da $F$. necrophorum rappresenta un evento piuttosto raro, gli anaerobi dovrebbero essere sempre considerati come potenziali responsabili di meningiti, soprattutto quando otiti o ascessi faringei, precedono o accompagnano l'inizio dei sintomi.

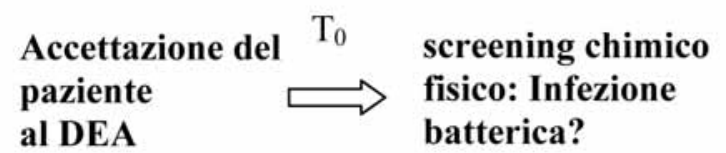

\section{4h emocoltura positiva: l'esame batteriosopico evidenzia bacilli gram negativi}

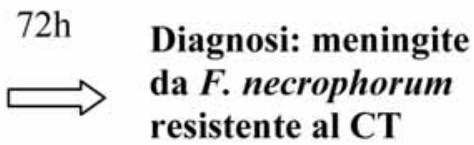

$6 \mathrm{dd}$

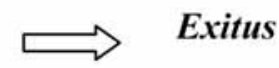

Figura I. Percorso diagnostico

\section{BIBLIOGRAFIA}

1. Chirinos JA, Lichtstein DM, Garcia J, Tamariz LJ. "The evolution of Lemierre syndrome: report of 2 cases and review of the literature" Medicine (Baltimore). 2002 Nov;81(6):458-65.
2. Karkos PD, Asrani S, Karkos CD, Leong SC, Theochari EG, Alexopoulou TD, Assimakopoulos AD. "Lemierre's syndrome: A systematic review" Laryngoscope. 2009 Aug;119(8):1552-9.

3. Brazier JS. "Human infections with Fusobacterium necrophorum". Anaerobe. 2006 Aug;12(4):165-72. Epub 2005 Dec 22. 